\title{
A Wireless and Real-Time Monitoring System Design for Car Networking Applications
}

\author{
Li Wenjun, Zhong Yiming, and Li Wenbin \\ Key Laboratory of RF Circuits and Systems of National MOE, Hangzhou Dianzi University, Hangzhou 310018, China \\ Correspondence should be addressed to Li Wenjun; liwenjun@hdu.edu.cn
}

Received 14 September 2013; Accepted 8 November 2013

Academic Editor: Guo Qing Luo

Copyright ( 2013 Li Wenjun et al. This is an open access article distributed under the Creative Commons Attribution License, which permits unrestricted use, distribution, and reproduction in any medium, provided the original work is properly cited.

\begin{abstract}
We described a wireless and monitoring system to obtain several classes of vehicle data and send them to the server via General Packet Radio Service (GPRS) in real-time. These data are consisted by on-board diagnostic (OBD) which get from the vehicle's OBD interface, Tire-Pressure Monitoring system (TPMS) and Global Positioning System (GPS). The main content of this paper is the hardware design of the system, especially RF modules and antennas.
\end{abstract}

\section{Introduction}

With the rapid development of automobiles, people need a more efficient way to manage cars. Sometimes we wish that not only drivers know the information about their cars, but also the administrator could do the same to all the cars which are the scope of his management in real-time. Even more we would like to store all the information data in the database so that we can analyze and check the history database, if needed. So the car networking has been put forward.Car networking means that we transform kinds of vehicle information into electronic singles, and we could exchange message with the outside communication systems, such as other cars, internet, and transport facilities [1]. In this way, we could manage the cars more effectively. However, car networking is just in its early stage since it could only communicate with internet. In this stage, car networking is mainly divided into three parts including data acquisition, data transmission, and data processing. Data acquisition and data transmission are implemented in in-vehicle terminal, as shown in Figure 1.

Many services which are based on Geographic Information System (GIS) are similar to this architecture, and supervisory system of school bus's track and the remote assistance system are examples of these services. They all need to upload the GPS data in real-time and store these data for check in the future [2]. However, if we could upload more vehicle information like tire pressure, engine information, oil mass of fuel tank, and diagnostic trouble codes, the control of the cars would be more powerful. It will enormously push forward the development of car networking application. Generally, people add sensors or other measuring equipments to obtain more information. But it is noteworthy that a car itself is an electronic system. Automobile manufacturers integrate a lot of sensors into the car before it leaves factory. From a computer science perspective, one car is a complex distributed real-time system with up to 60 microcontroller based electronic control units (ECUs) that communicate via approximately 2500 different signals with each other [3]. And fortunately, we can have access to those data via a special interface called OBD. OBD stands for "on-board diagnostics," a computer-based system built into all model year (MY) 1996 and newer lightduty cars and trucks. OBD monitors the performance of some of the engines' major components, including individual emission controls. The system provides owners with an early warning of malfunctions by way of a dashboard "Check Engine" light (also known as a Malfunction Indicator Light or MIL, for short). By giving vehicle owners this early warning, OBD protects not only the environment but also consumers, identifying minor problems before they become major repair bills [4]. We can get the current operational status of the car or the diagnostic trouble codes by sending commands in accordance with a predetermined communication protocol 


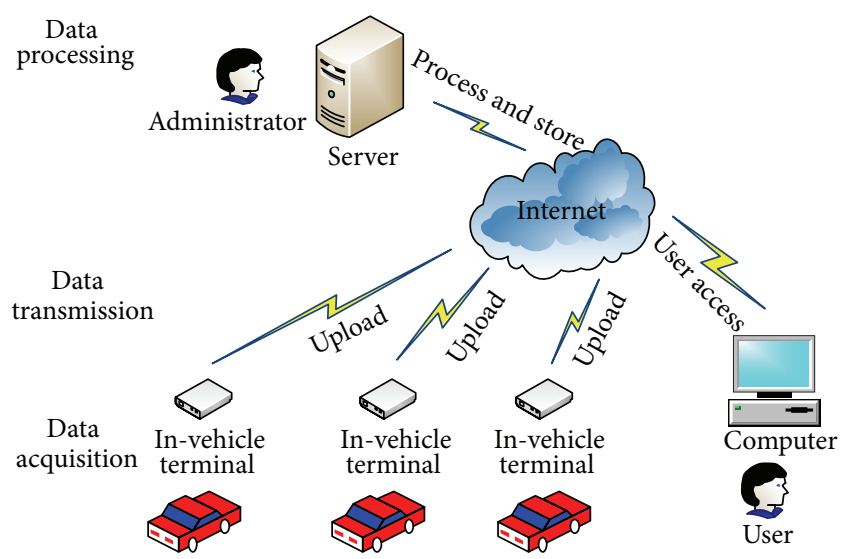

FIGURE 1: Car networking architecture in early stage.

standard [5]. The system introduced in this paper takes full advantages of the functionality provided by OBD. As referring to the international standards ISO15031-5 and ISO14230, the system could be compatible with most cars [6].

\section{Design of In-Vehicle Terminal}

The in-vehicle terminal is comprised of Microprogrammed Control Unit (MCU), Single chip OBD protocol converter, GPS module, GPRS module, and RF receiver chip. Its hardware design and the function modules are shown in Figure 2.

Even though OBD have the unified standard of application layer, there are different physical layers and network layers from different manufacturers. In order to be compatible with more manufacturers and increase the stability of the system, we choose single chip protocol converter TL718 as the protocol converter between MCU and the car's OBD interface. TL718 supports up to 16 kinds of OBD physical layers, so it could meet the requirement completely. TL718, GPS module and GPRS module are all connected with MCU via serial port, and the communication between RF module and MCU is based on a single data line. After receiving all data from TL718, GPS, and RF receiver chip, MCU will encapsulate those data as one data packet and sent it to GPRS module, then it uploaded it to server.

As we can see, the TPMS's communication, GPS data reception and GPRS data transmission all involve wireless communication, so we dig into the discussion on how to optimize the choice of those modules and which antennas which to match those modules to.

2.1. Wireless Tire Pressure Data Acquisition for Car. Some data show that there are more and more car accidents which are caused by tire burst, especially when a car is running at a high speed in summer. In most cases, the tire is very critical for the safety of the running automobile. Now, the most effective way to prevent tire burst is to monitor the temperature and pressure of the tires.

As we know, the sensor for tire pressure monitoring is installed in the tire, while the module for receiving tire pressure data should be placed within the carriage, considering that the tire is a relatively independent unit which is located outside the carriage. Moreover, it is in the high-speed rotating state when the car is running. Thus the only way to realize the communication of real-time tire pressure monitoring system is to use the wireless communication technology.

Two of the commonest frequencies used in RF communication are $433 \mathrm{MHz}$ and $2.4 \mathrm{GHz}$. Compared to $433 \mathrm{MHz}$, $2.4 \mathrm{GHz}$ frequency band has a lot of advantages, such as fast communication rate, good safety and convenient protocol extension. However, our system does not need a high communication rate, because the kinds of data about tire are only temperature and pressure, and they all change at a relatively slow rate (checked once per half minute). Besides, there are a limited number of tires in one car, so we can set up a simple communication network by an easy protocol.

Moreover, the higher the frequency of the electromagnetic wave, the faster the attenuation. And the attenuation is more apparent, as the main material of car is metal. With comprehensive consideration, $433 \mathrm{MHz}$ frequency is a better choice, which can be used easily, and the diffraction is better as its wavelength is longer [7].

Although there are many types of transceiver chip in $433 \mathrm{MHz}$ frequency band, considering the actual application that the tire pressure sensor works in a single launching state and the car terminal works in a single receiving state, we choose independent single launching and receiving chip to save costs and bring down power consumption. From the application perspective, the low power consumption and stable performance are the most critical factors in the TPMS system then we find that launching chip MICRF112 and receiving chip MICRF211 of Micrel company can fulfill our requirement.

Next, we need to select the matching antenna for the RF chip. According to antenna theory, when the antenna length is proportional to the wavelength of the electromagnetic wave, sending and receiving efficiency will be the highest. This brings up the question if we chose the diffraction better and longer wavelength frequency band, it also means that we choose a longer antenna. At the same time, considering the rotation of the tire, omnidirectional antenna is more suitable antenna selection. As a result, we select helical antenna for the tire pressure sensor. According to the antenna theory, as the ratio of the wavelength and the diameter of the helical antenna $D / \lambda$ meet $D / \lambda<0.18$, the antenna radiation characteristics will be an omnidirectional radiation antenna. As the wavelength of $433 \mathrm{MHz}$ band is $69 \mathrm{~cm}$ and the diameter of sensor is $1 \mathrm{~cm}$, the ratio is given by $D / \lambda=1 / 69=0.01<$ 0.18 which quite satisfies the inequality. This way, we can make an omnidirectional radiation antenna for RF module working in $433 \mathrm{MHz}$, and meanwhile the antenna is limited in a finite dimension [8].

2.2. GPS Data Acquisition Module. For all kinds of car networking applications, GPS information is almost essential information. Because the location for moving objects is one of the most critical attributes, so, we need to improve GPS signal quality as much as possible. 


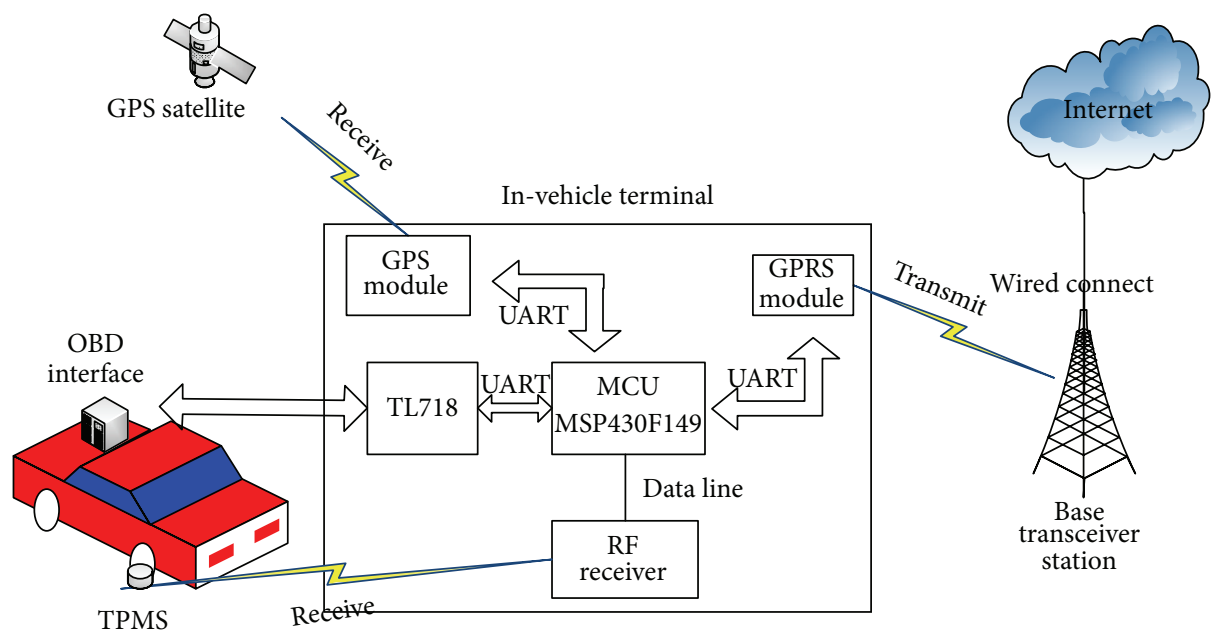

FIGURE 2: Hardware design structure and function of the terminal.

In the case of the original GPS design, two frequencies are utilized; one at $1575.42 \mathrm{MHz}$ called L1; and a second at 1227.60 MHz called L2. And, in order to eliminate the Faraday rotation effect of the ionosphere to signal, GPS signal is a right-handed circularly polarized, so receiving antenna is also the right circular polarization mode [9].

Now, we consider two GPS antennas that are commonly used, patch antenna and helical antenna. Researches indicated that it is particularly difficult to achieve the crosspolarization rejection ratio and also to achieve adequate impedance matching at both the L1 and L2 frequencies using patch antenna. Patch antennas are narrow band, and a special feed is required to achieve the desired circular polarization. However, the study on the design based on an array of square helical antennas showed that the values of cross polarization rejection patterns slope satisfy the requirements to design a high-performance GPS receiving antenna [10]. Besides, the GPS client must receive the satellite signal directly, so the signal quality of a GPS receiver is generally weak.

Taking all above factors into account, we choose the helical antennas with a low noise amplifier. In order to reduce the signal attenuation, we extend the length of the antenna and place the receiver close to the window in the same time.

2.3. GPRS Module. The only way to send the car's information data to server is via wireless communication. Currently, there are multiple ways to construct wireless communication system, which are mainly divided into two classes, building their own private network and directly accessing to the public network. The first way, building their own private network, can significantly improve the communication efficiency and enrich vehicular networking functions. However, the biggest problem is the huge upfront cost to build communication infrastructure. Therefore, most of the prophase vehicular networking programs choose the second way, directly accessing to the public network. The greatest advantage of this way is that initial investment is lower and it can quickly come into use. Besides, another benefit of this way is that it has a large coverage area.
As we all know, there are two types of public communication technology, 2G like GSM and 3G like WCDMA. Compared with each other, the coverage area of GSM network is very wide because GSM technology has developed for so many years, but the disadvantage of GSM is that the bandwidth is small and the relative cost is high. $3 \mathrm{G}$ is the most advanced communication technology, which has been used. It greatly increases the communication bandwidth, so it is suitable for the transmission of large amounts of data, such as real-time video information. However, its main disadvantage is the limited network coverage area. Currently, 3G signal only covers the downtown with a lot of crowd. This limits the use scope of $3 \mathrm{G}$ communication systems.

The system described in this paper aims at vehicles which do not just move among densely populated urban. However, there are many areas without $3 \mathrm{G}$ signal, such as the lonely stretch of highway. At present, the information monitored by the system is only some character data, no images or video information. The bandwidth requirement is small but it requires a larger network coverage area. Therefore, the final selection of the system is GPRS. The system adopts SIM900A module of SIMcom company which uses SMT package and compact size of GSM/GPRS single-chip solution. This module has been maturely used in many fields. SIM900A connected with the MCU via the serial port receives the command and data packets sent by the MCU and then send them to the specified internet address.

GPRS operates at three frequency bands, $900 \mathrm{MHz}$, $1800 \mathrm{MHz}$, and $1900 \mathrm{MHz}$. The antennas of GPRS are called Flexible Printed Circuit Board (FPCB) Antennas which are frequently used in mobile phones and other mobile devices at present. FPCB antenna has wide bandwidth which is enough to support all three frequency bands of GPRS, as well as flexible and variable forms. It is easy to be integrated into a variety of terminal equipment [11].

As GPRS communications just need connecting with nearby base stations (much easier than GPS communications which have to connect directly with satellites), the quality of the communication signal is generally good. 
TABLE 1: Frame Format of Frame's Header is "AM".

(a)

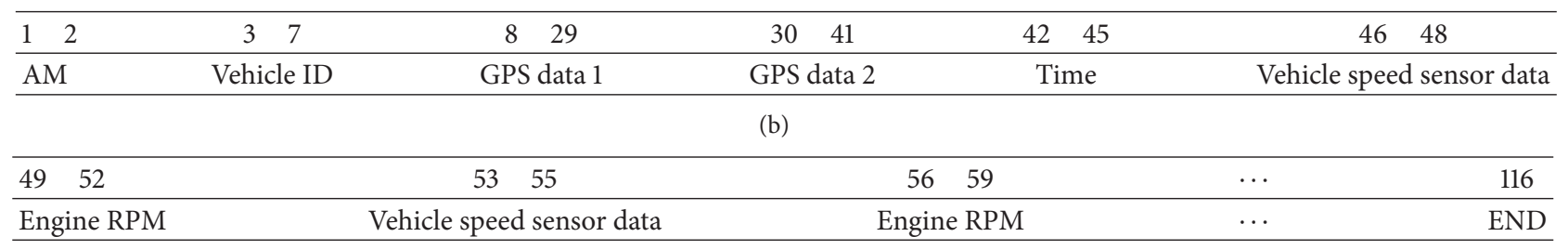

TABle 2: Frame Format of Frame's Header is "BM".

(a)

\begin{tabular}{|c|c|c|c|c|c|}
\hline 2 & 37 & 29 & $30 \quad 41$ & $42 \quad 45$ & 46 \\
\hline $\mathrm{BM}$ & Vehicle ID & GPS data 1 & GPS data 2 & Time & Engine coolant temperature \\
\hline
\end{tabular}

(b)

\begin{tabular}{|c|c|c|c|}
\hline 51 & $52 \quad 54$ & 55 & 60 \\
\hline Battery voltage & Intake air temperature & Distance travelled while MIT is activated & END \\
\hline
\end{tabular}

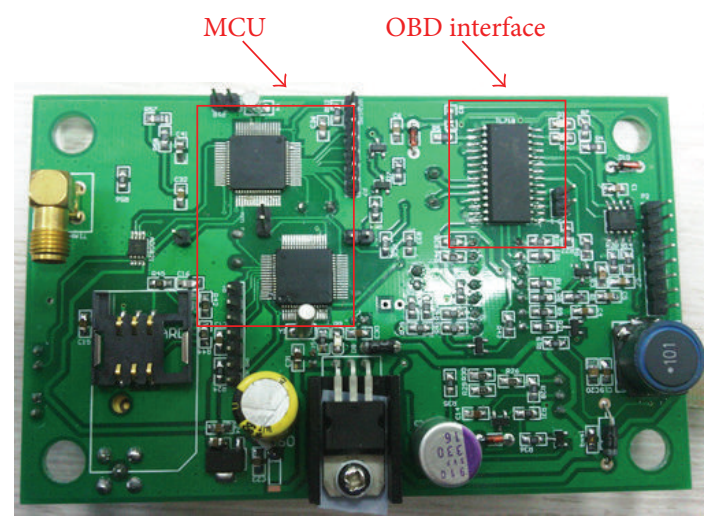

FIgURE 3: The top view of the terminal.

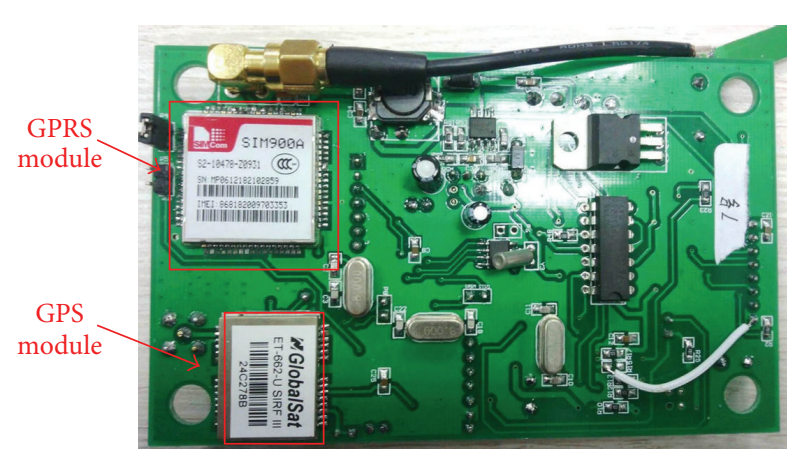

FIGURE 4: The reverse side view of the terminal.

The server will receive three kinds of data packets, each of which has different frame formats.

In the end, the power of the in-vehicle terminal is supplied by OBD interface which outputs $12 \mathrm{~V}$ DC. Then the pictures of the in-vehicle terminal are shown in Figures 3 and 4.

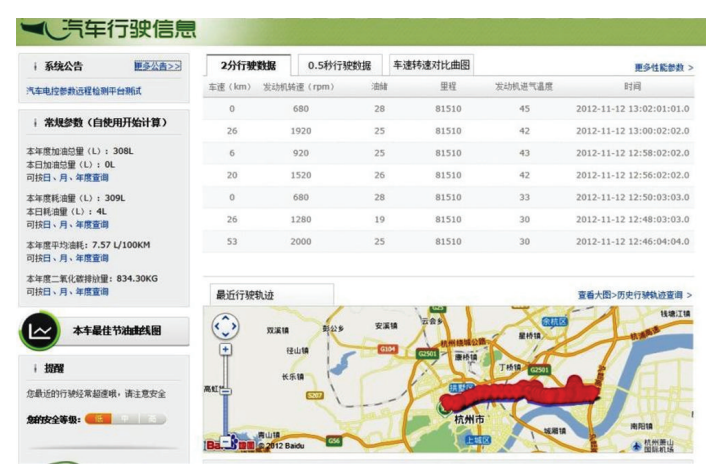

FIGURE 5: User interface via a browser of the system.

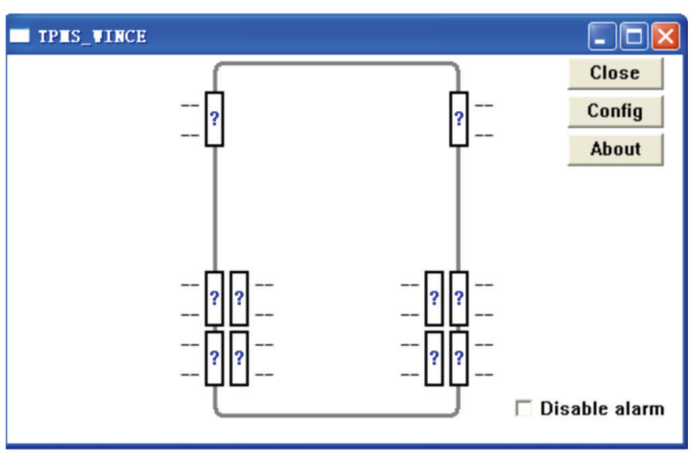

FIGURE 6: Software interface of TPMS data.

\section{Data Processing and Result}

The frame header "AM," as shown in Table 1, means that this frame has Vehicle Speed Sensor data and Engine RPM data which read twice per second. But the frame is sent per five second so that we could make good use of GPRS's data traffic. 
TABle 3: Frame Format of Frame's Header is "CM".

(a)

\begin{tabular}{|c|c|c|c|c|c|}
\hline 2 & 7 & 29 & 30 & $42 \quad 45$ & 46 \\
\hline $\mathrm{CM}$ & Vehicle ID & GPS data 1 & GPS data 2 & Time & Tire A1 pressure \\
\hline
\end{tabular}

(b)

\begin{tabular}{|c|c|c|c|c|}
\hline $50 \quad 52$ & 57 & 58 & $\cdots$ & 74 \\
\hline Tire A1 temperature & Tire A2 pressure & Tire A2 temperature & $\ldots$ & END \\
\hline
\end{tabular}

The frame header "BM," as shown in Table 2, means that this frame has some data which change slowly, so those data are read only once per five minute.

The frame header "CM," as shown in Table 3, means that this frame has the TPMS data.

Based on this data, user could check the car's information via a browser to access the server. People can review the history vehicle track and the running state of the car, as shown in Figure 5, or the car's tires pressure and temperature, as shown in Figure 6.

\section{Conclusion}

The results successfully show that the system can acquire and transmit a lot of imported data about vehicle. As making the most of those vehicle information, this system has broad prospects. This system can play the roles of Black Box for car. The running state data are very useful to analyze traffic incidents causation then it can be applied to insurance claims processing. And it could also be used in remote assistance system, by analyzing the diagnostic trouble codes.

\section{Acknowledgment}

The authors are thank for the help provided by the "The Key Technology And Application of Intelligent Identification" Priority Scientific and Technological Innovation Team from Zhejiang province (2010R50010).

\section{References}

[1] G. Karagiannis, O. Altintas, E. Ekici et al., "Vehicular networking: a survey and tutorial on requirements, architectures, challenges, standards and solutions," IEEE Communications Surveys and Tutorials, vol. 13, no. 4, pp. 584-616, 2011.

[2] Y.-H. Chou, "Automatic bus routing and passenger geocoding with a geographic information system," in Proceedings of the 6th Vehicle Navigation and Information Systems Conference, pp. 352-359, Seattle, Wash, USA, August 1995.

[3] T. Steinbach, F. Korf, and T. C. Schmidt, "Real-time Ethernet for automotive applications: a solution for future in-car networks," in Proceedings of the 1st IEEE International Conference on Consumer Electronics (ICCE '11), pp. 216-220, Berlin, Germany, September 2011.

[4] H. J. Yun, S. K. Lee, and O. C. Kwon, "Vehicle-generated data exchange protocol for Remote OBD inspection and maintenance," in Proceedings of the International Conference on
Computer Sciences and Convergence Information Technology (ICCIT '11), pp. 81-84, Seogwipo, Republic of Korea, 2011.

[5] L. E. Cordova-Lopez, A. Mason, J. D. Cullen, A. Shaw, and A. I. Al-Shamma'A, "Online vehicle and atmospheric pollution monitoring using GIS and wireless sensor networks," Journal of Physics, vol. 76, no. 1, Article ID 012019, 2007.

[6] International Organization for Standardization, "Road vehicles-Communication between vehicle and external equipment for emissions-related diagnostics-part 5: emissions-related diagnostic services," ISO, ISO15031-5, 2006.

[7] L. Shang and W. F. Li, "Choice of optimal frequency for radio orientation system in mine tunnel," Safety in Coal Mines, vol. 39, no. 3, pp. 22-25, 2008.

[8] Y. Suo and J. H. Qiu, "Analysis and design of normal mode helical antenna," Equipment Environmental Engineering, vol. 5, no. 1, pp. 81-83, 2008.

[9] K. Phaebua, C. Phongcharoenpanich, D. Torrungrueng, N. Surittikul, and W. Villarroel, "Dual-band antenna system for SDARS and GPS applications," in Proceedings of the IEEE Antennas and Propagation Society International Symposium (APSURSI '10), pp. 1-4, Toronto, Canada, July 2010.

[10] J. I. Ortigosa, N. Padros, and M. F. Iskander, "Comparative study of high-performance GPS receiving antenna designs," in Proceedings of the Antennas and Propagation Society International Symposium, vol. 3, pp. 1958-1961, Baltimore, Ma, USA, 1996.

[11] X. Zhang and A. Zhao, "Flexible compact planar invertedF antenna for GSM/DCS/PCS triple-band applications," in Proceedings of the 8th International Symposium on Antennas, Propagation and EM Theory (ISAPE '08), pp. 15-18, November 2008. 

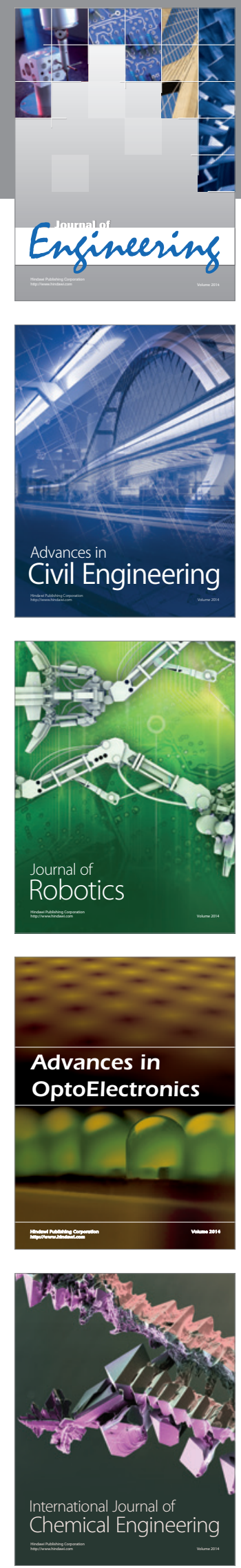

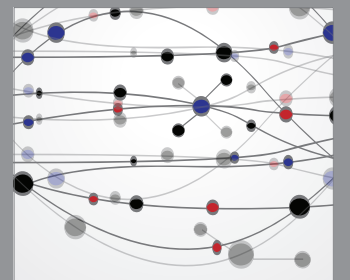

The Scientific World Journal
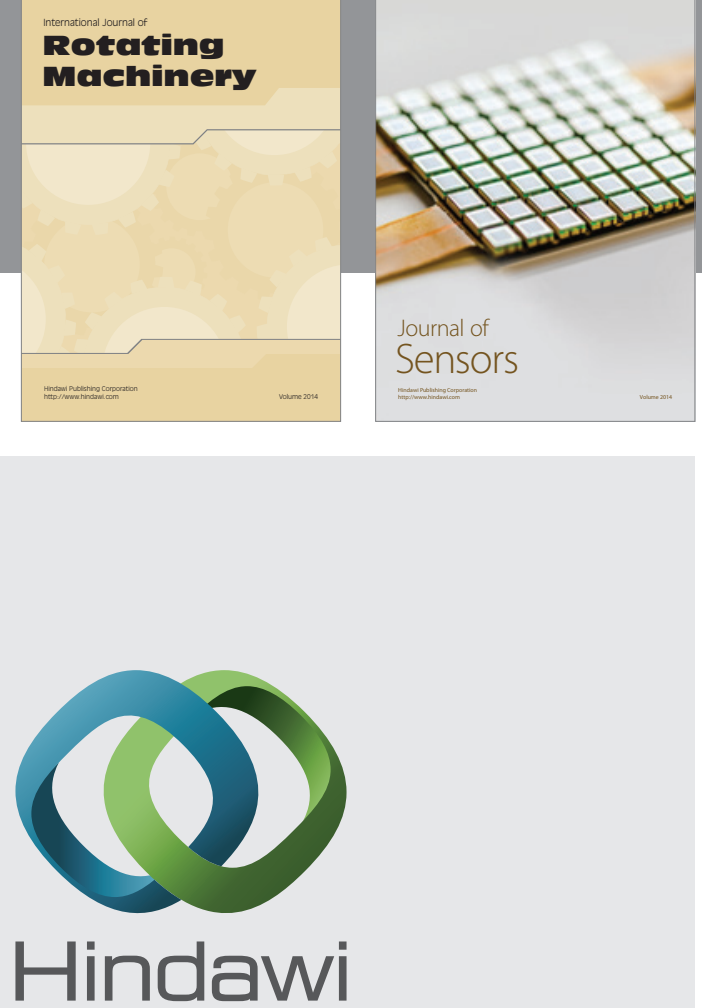

Submit your manuscripts at http://www.hindawi.com
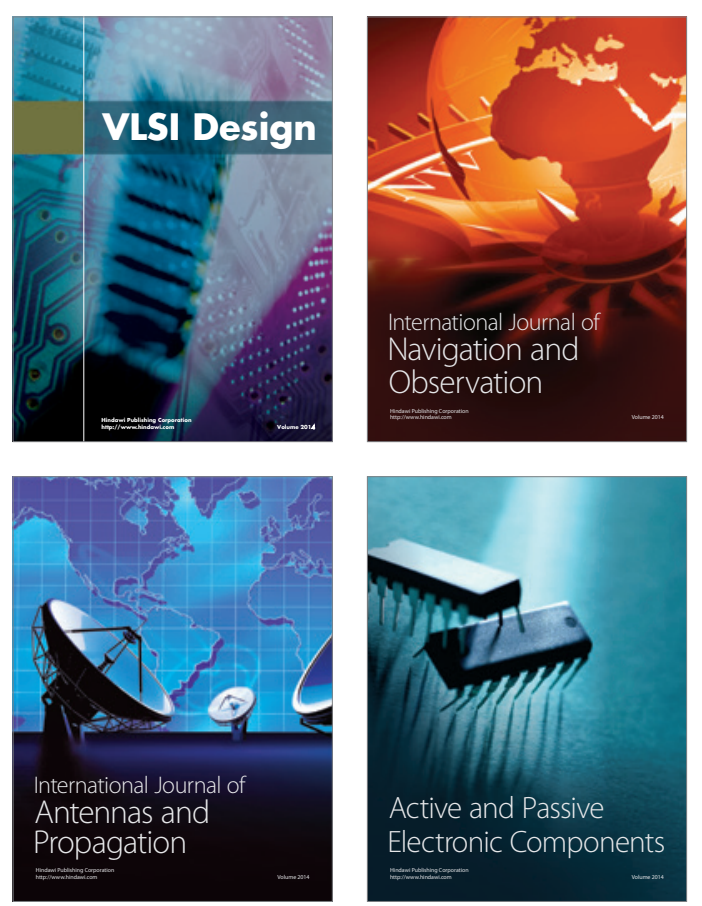
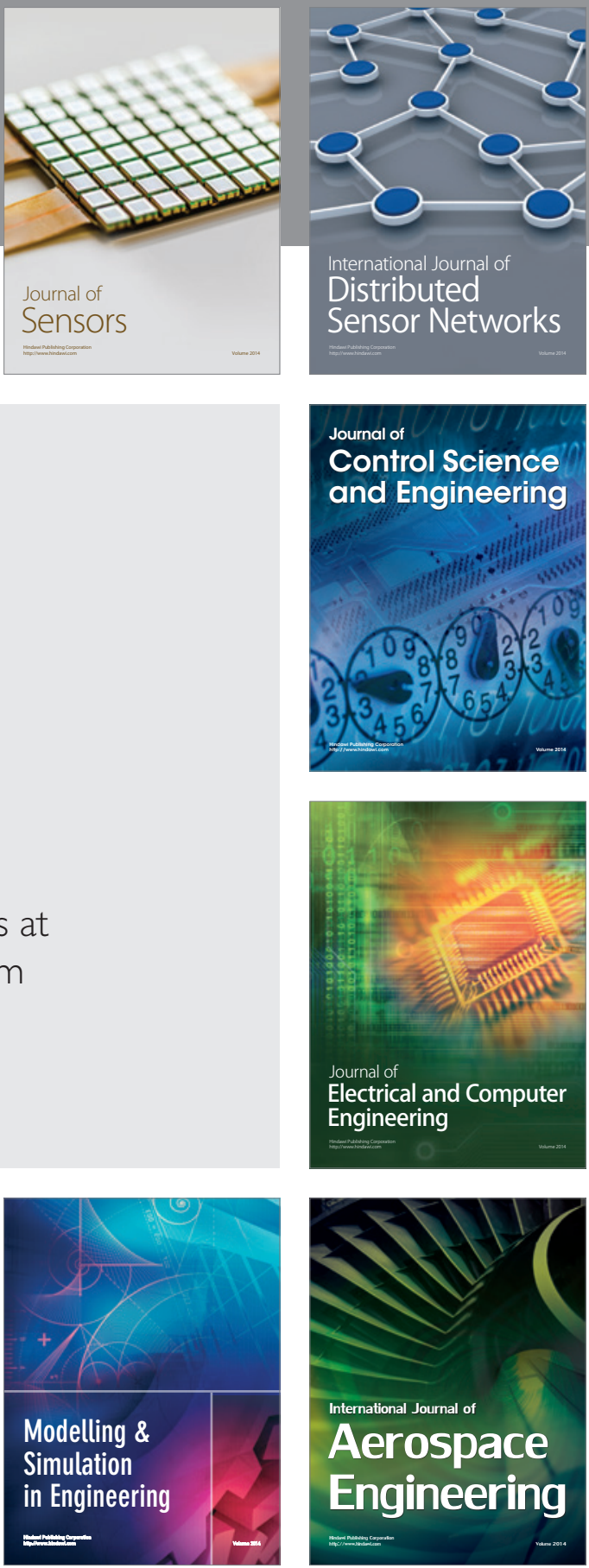

Journal of

Control Science

and Engineering
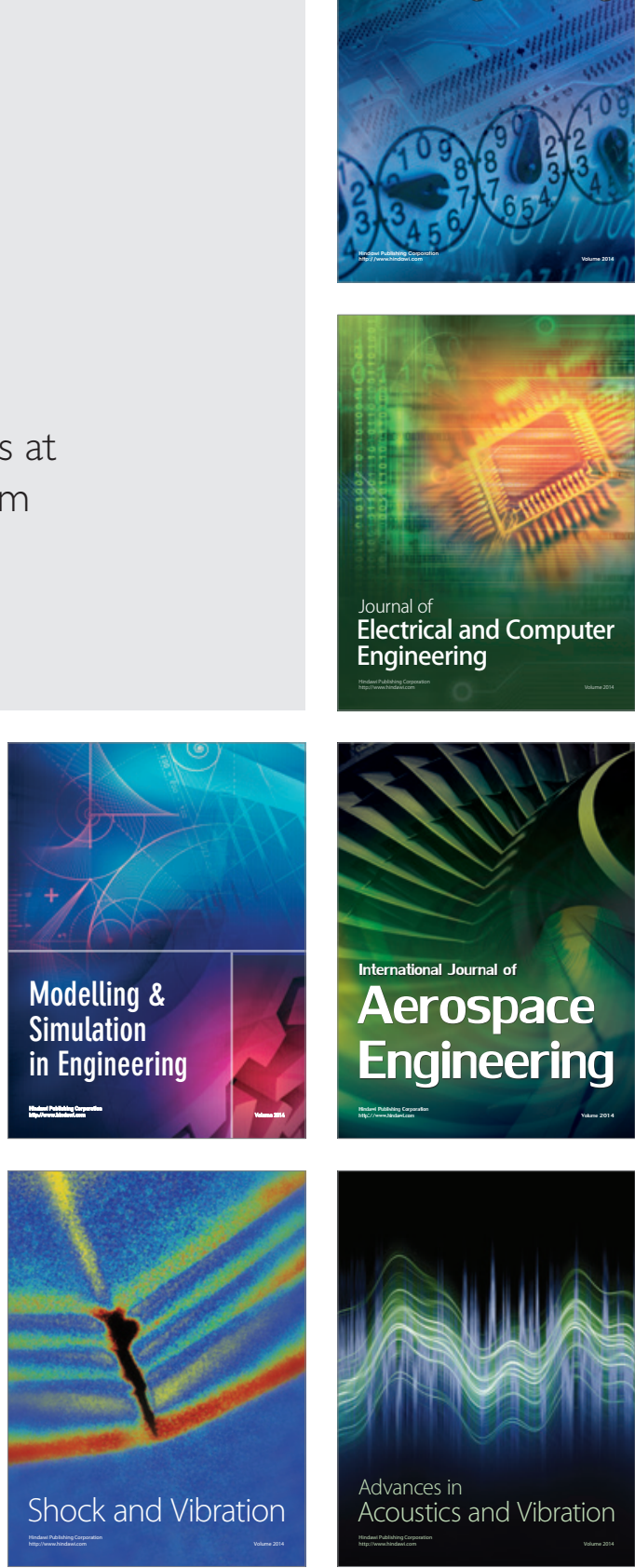